\title{
Video Article \\ Specimen Preparation, Imaging, and Analysis Protocols for Knife-edge Scanning Microscopy
} \author{
C. Abbott ${ }^{5}$ \\ ${ }^{1}$ Department of Computer Science and Engineering, Texas A\&M University \\ ${ }^{2}$ Beckman Institute for Advanced Science and Technology, University of Illinois \\ ${ }^{3}$ Department of Electrical and Computer Engineering, Kettering University \\ ${ }^{4} 3$ Scan \\ ${ }^{5}$ Department of Veterinary Integrative Biosciences, Texas A\&M University
}

Yoonsuck Choe ${ }^{1}$, David Mayerich ${ }^{2}$, Jaerock Kwon ${ }^{3}$, Daniel E. Miller ${ }^{1}$, Chul Sung ${ }^{1}$, Ji Ryang Chung ${ }^{1}$, Todd Huffman ${ }^{4}$, John Keyser ${ }^{1}$, Louise

Correspondence to: Yoonsuck Choe at choe@tamu.edu

URL: https://www.jove.com/video/3248

DOI: doi:10.3791/3248

Keywords: Bioengineering, Issue 58, Physical sectioning, serial sectioning, light microscopy, brain imaging, microtome

Date Published: 12/9/2011

Citation: Choe, Y., Mayerich, D., Kwon, J., Miller, D.E., Sung, C., Chung, J.R., Huffman, T., Keyser, J., Abbott, L.C. Specimen Preparation, Imaging, and Analysis Protocols for Knife-edge Scanning Microscopy. J. Vis. Exp. (58), e3248, doi:10.3791/3248 (2011).

\section{Abstract}

Major advances in high-throughput, high-resolution, 3D microscopy techniques have enabled the acquisition of large volumes of neuroanatomical data at submicrometer resolution. One of the first such instruments producing whole-brain-scale data is the Knife-Edge Scanning Microscope (KESM), 5, , developed and hosted in the authors' lab. KESM has been used to section and image whole mouse brains at submicrometer resolution, revealing the intricate details of the neuronal networks (Golgi) ${ }^{1,4,8}$, vascular networks (India ink) $)^{1,4}$, and cell body distribution (Nissl) ${ }^{3}$. The use of KESM is not restricted to the mouse nor the brain. We have successfully imaged the octopus brain ${ }^{6}$, mouse lung, and rat brain. We are currently working on whole zebra fish embryos. Data like these can greatly contribute to connectomics research ${ }^{10}$; to microcirculation and hemodynamic research; and to stereology research by providing an exact ground-truth.

In this article, we will describe the pipeline, including specimen preparation (fixing, staining, and embedding), KESM configuration and setup, sectioning and imaging with the KESM, image processing, data preparation, and data visualization and analysis. The emphasis will be on specimen preparation and visualization/analysis of obtained KESM data. We expect the detailed protocol presented in this article to help broaden the access to KESM and increase its utilization.

\section{Video Link}

The video component of this article can be found at https://www.jove.com/video/3248/

\section{Protocol}

\section{Specimen preparation: Golgi-Cox}

1. This protocol closely follows the protocol of Mayerich et al. ${ }^{7}$.

2. The C57BL/6J mouse is deeply anesthetized using isoflurane inhalant anesthesia and then decapitated.

3 . The brain is removed and placed into a Golgi-Cox fixation solution ( $1 \%$ potassium chromate, $1 \%$ potassium dichromate, and $1 \%$ mercuric chloride in deionized water).

4. The brain is left in the Golgi-Cox solution in the dark, at room temperature, for 10 to 16 weeks.

5. The brains are rinsed in deionized water overnight in the dark.

6. The rinsed brain is immersed in $5 \%$ ammonium hydroxide solution in deionized water for 7 to 10 days in the dark at room temperature. This lengthy preparation time was to ensure infiltration of the entire brain, so that the black precipitate had a chance to completely form in the tissue.

7. The brain is rinsed again in deionized water at room temperature for 4 hours and then dehydrated through a graded series of ethyl alcohols: $50 \%$ and $70 \%$ in the refrigerator $\left(4{ }^{\circ} \mathrm{C}\right.$ ), and $85 \%, 95 \%$ (3 changes), and $100 \%$ (4 to 5 changes) in room temperature. The brain is left in each solution for 24 hours.

8. The dehydrated brain is then put in acetone ( 3 to 4 changes, each for one day), followed by an araldite-acetone mixture with araldite-toacetone ratio of $1: 2,1: 1,2: 1$, and finally in $100 \%$ araldite (3 changes, each time overnight), in the refrigerator $\left(4^{\circ} \mathrm{C}\right)$.

9. Finally, the treated brain is embedded in $100 \%$ araldite and heated to $60{ }^{\circ} \mathrm{C}$ for 3 days (cf. embedding protocol in Abbott and Sotelo ${ }^{2}$ ). If specimens are embedded in LR White then the specimens are transferred from the last $100 \%$ ethanol solution through three changes of LR White solution that are each kept in the refrigerator overnight, which is a standard procedure prior to polymerization. Three changes are done 
to ensure full infiltration. The specimen is then transferred to fresh LR White that is polymerized in a closed container at $60{ }^{\circ} \mathrm{C}$ for 24 hours, which is the required amount of time and temperature for proper polymerization.

10. Fig. 1 shows a Golgi-Cox stained brain embedded in Araldite.

11. The cured specimen block is then mounted on the metal specimen ring using epoxy, and the sides of the block trimmed as necessary (see Fig. 2).

\section{Specimen preparation: NissI}

1. This protocol closely follows the protocol of Mayerich et al. ${ }^{7}$

2. The mouse is deeply anaesthetized using ketamine and xylazine injected intraperitoneally and then perfused transcardially using $50 \mathrm{~mL}$ of

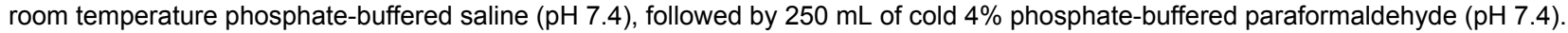

3. The mouse is then perfused with $100 \mathrm{~mL}$ of a solution of $0.1 \%$ thionin dye in deionized water, and the body placed in the refrigerator $\left(4{ }^{\circ} \mathrm{C}\right)$ for 24 hours.

4. After 24 hours, the brain is removed from the calvaria and placed in a fresh solution of $0.1 \%$ thionin and left at $4{ }^{\circ} \mathrm{C}$ for 7 days. The lengthy duration of this procedure was to ensure full infiltration of thionin into the en bloc preparation.

5. The brain is then destained and dehydrated through a graded series of ethanols starting with $50 \%$ ethanol and water and increasing to $100 \%$ ethanol over a time period of 6 weeks. The ethanol changes from $50 \%$ to $70 \%$ are stored in the refrigerator $\left(4{ }^{\circ} \mathrm{C}\right)$ and all changes of ethanol above $70 \%$ are stored at room temperature.

6. After three changes of acetone (2-4 days in each solution at room temperature), the brain is then embedded in araldite plastic following the protocol in 1.8-1.9 above. If LR white is the embedding medium then the protocol in 1.10 above is followed.

\section{Specimen preparation: India ink}

1. The mouse is deeply anaesthetized using ketamine and xylazine ( $1.7 \mathrm{mg}$ ketamine / $0.26 \mathrm{mg}$ xylazine per 20 grams body weight) injected intraperitoneally and then perfused transcardially using $50 \mathrm{~mL}$ of room temperature phosphate-buffered saline (pH 7.4), followed by $250 \mathrm{~mL}$ of room temperature $10 \%$ neutral buffered formalin $(\mathrm{pH} 7.4)$. and finally with $3.0 \mathrm{cc}$ of undiluted India ink.

2. Whole body perfusion with saline and fixative is necessary to clear the blood from the cardiovascular system and to fix the tissues. Perfusion with India ink is necessary to completely fill the vasculature of the cardiovascular system.

3. The resulting brain is then dehydrated through a series of graded ethyl alcohols $(25 \%-100 \%)$ and then embedded in araldite plastic following the protocol in 1.8-1.9 above. If LR white is the embedding medium then the protocol in 1.10 above is followed.

\section{Specimen preparation: Generic species, generic organs}

1. For the generic case, fixation can be done with either $10 \%$ neutral buffered formalin or $4 \%$ paraformaldehyde.

2. For small tissue volumes, diffusion instead of perfusion is recommended for fixation and staining. In the case of small organisms or organs, staining also can be done during the dehydration process.

3. The embedding protocol is the same as above, although the times for solution infiltration can be reduced for organs or organisms that are much smaller than whole mouse brain.

\section{KESM setup and imaging}

1. Turn off pump, turn on stage, turn on camera, turn on illuminator.

2. Raise knife and objective.

3. Install specimen ring.

4. Measure specimen dimension and create a configuration file for the KESM Stage Controller2 application.

5. Start KESM Stage Controller2 and initialize stage.

6. Lower knife/objective, turn on pump.

7. Focus objective and camera, by turning the focusing knob on the optical train and adjusting the camera's field of view relative to the knife edge.

8. Press on [Go] to initiate imaging.

9. See Figs. 3-8. See ${ }^{9,7}$ for technical details.

\section{Image processing and data preparation}

1. Copy the KESM Stack Processor executable into the data folder under the configuration file folder (00000, 00001, etc.).

2. Run the KESM Stack Processor to remove lighting artifact and normalize the background intensity in all images. Repeat for all data subfolders.

3. Prepare multiscale tiles from the data set and upload and set up the files in the KESM Brain Atlas web server.

4. See Fig. 9.

\section{Data visualization and analysis}

1. Visualization using the KESM Brain Atlas. Go to http://kesm.cs.tamu.edu and select the appropriate atlas.

1. Navigate as in Google maps.

2. Zoom in and out as in Google maps. 
3. To go to a different depth, select how deep to go at each step from the pull-down menu, and then click on either [+] or [-] to increase or decrease $z$ depth.

4. Adjust the overlay number by using the pull-down menu.

5. Adjust the overlay interval by using the pull-down menu.

6. See Fig. 11.

2. Visualization using MeVisLab.

1. Launch MeVisLab.

2. Create a new project.

3. In the following, new modules can be creating by typing in the module name in the command box.

4. Create module [Compose3Dfrom2DFiles], and go to the desired folder. Enter file string and click in [GetFileList]. Make sure the selected images can fit in memory. Click [Create3D] to create volume.

5. Create module [SetWorldMatrix], and set the "Scale" under "Elementary Transforms" $x, y, z$ to the appropriate voxel size (0.6, 0.7, 1.0 for a 10X 0.45NA objective). Link [Compose3Dfrom2DFiles] to [SetWorldMatrix].

6. Set Matrix and Transforms under "Matrix Composition" to "Ignore", "Ignore", "Forward".

7. Create module [Arithmetic1] and set the "Function" to "Invert(Max-Img)". Link [SetWorldMatrix] to [Arithmetic1].

8. Create module [View3D] and connect [Arithmetic1]

9. In View3D, while right mouse button is pressed, move the mouse around to adjust the threshold. You can crop regions, change orientation (move mouse while left mouse button is pressed), change illumination (volume rendering or MIP), turn on/off background, etc.

10. See Fig. 10.

\section{Representative Results:}

Here, we present whole-brain data and details. Figs. 12-15 show whole-brain India Ink, Golgi, and Nissl data sets ${ }^{1,4,3}$.

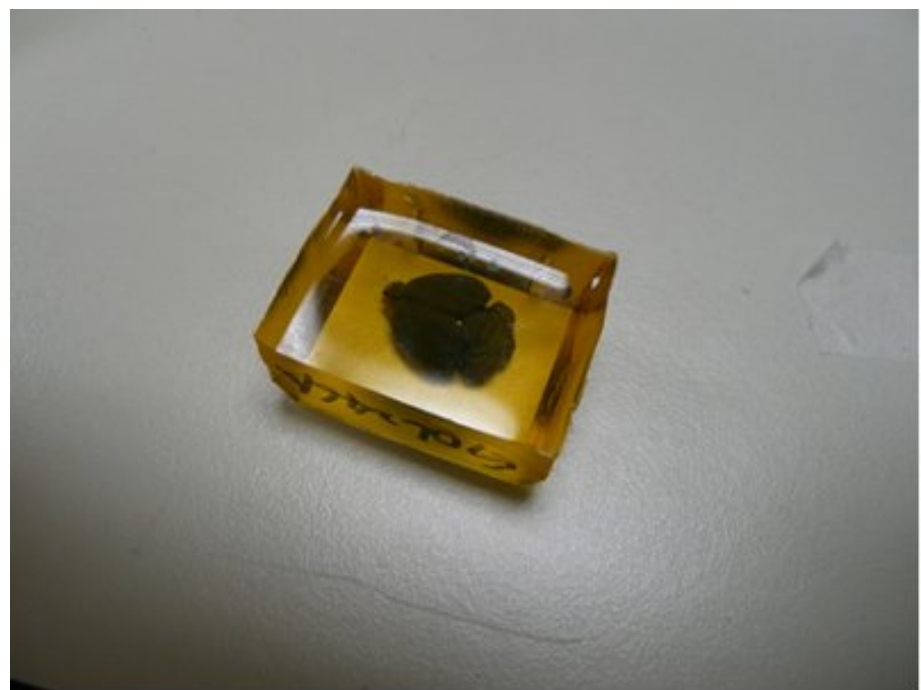

Figure 1. Fixed, stained, and embedded mouse brain 


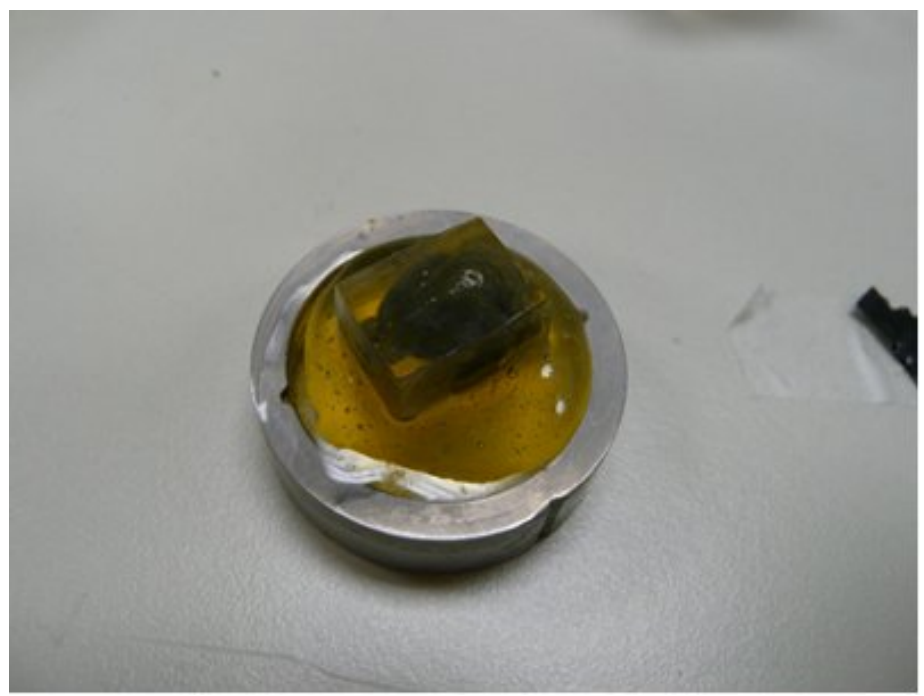

Figure 2. Embedded mouse brain specimen mounted on the specimen ring.

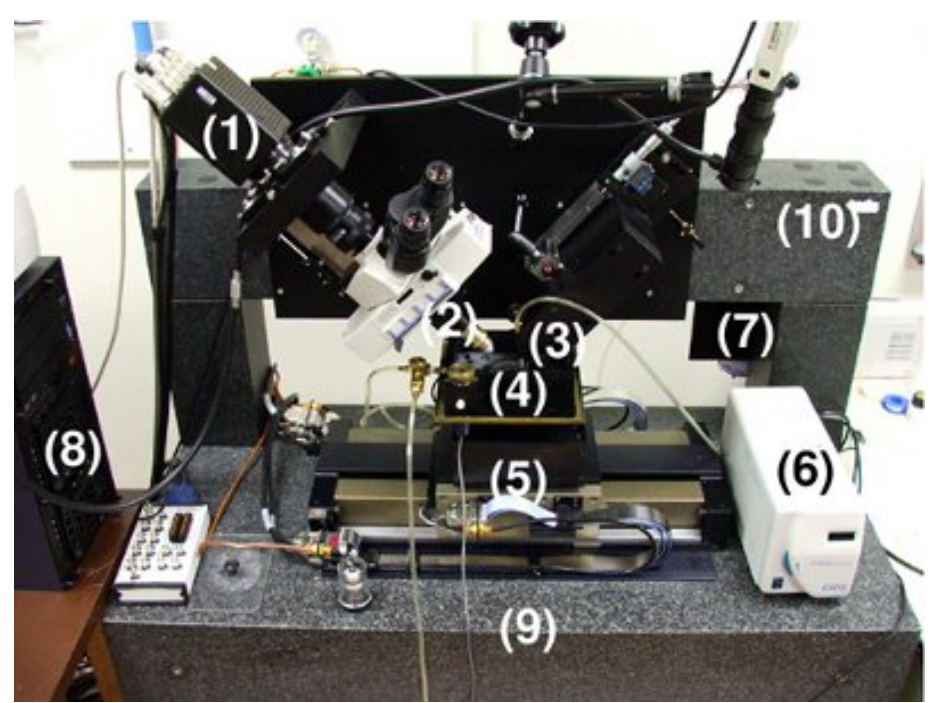

Figure 3. The Knife Edge Scanning Microscope (KESM). A photo of the KESM is shown with its major components marked: (1) high-speed linescan camera, (2) microscope objective, (3) diamond knife assembly and light collimator, (4) specimen tank (for water immersion imaging), (5) three-axis precision air-bearing stage, (6) white-light microscope illuminator, (7) water pump (in the back) for the removal of sectioned tissue, (8) PC server for stage control and image acquisition, (9) granite base, and (10) granite bridge.

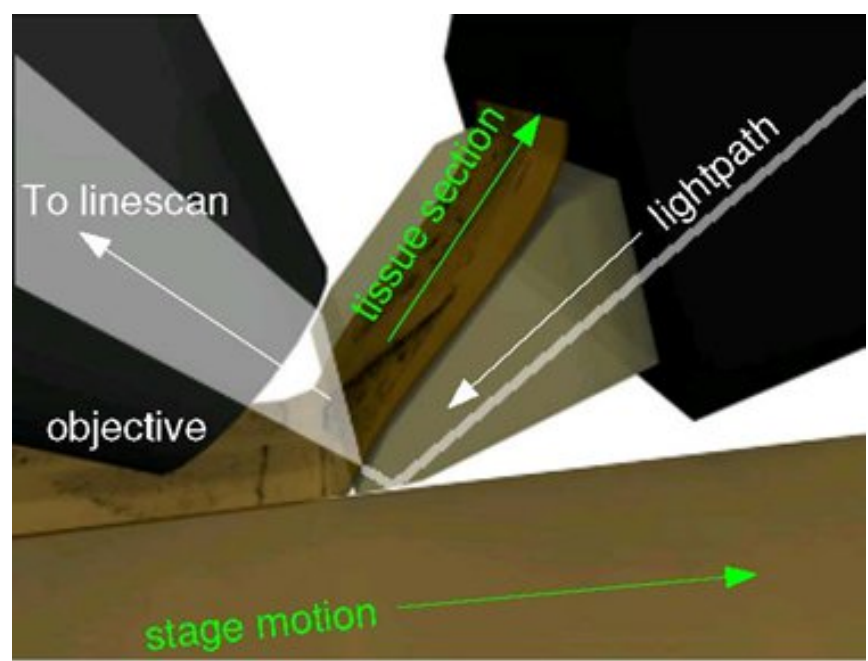

Figure 4. Imaging principles of the KESM. The principal of operation of KESM is illustrated. The objective and the knife is held in place, while the specimen affixed on the positioning stage moves (arrow with solid line) at the resolution of $20 \mathrm{~nm}$ and travel speed of 1-5, and gets scraped 
against the diamond knife ( $5 \mathrm{~mm}$ wide for 10X objective), generating a thin section flowing over the knife (arrow with solid line). Line-scan imaging is done near the very tip of the knife.

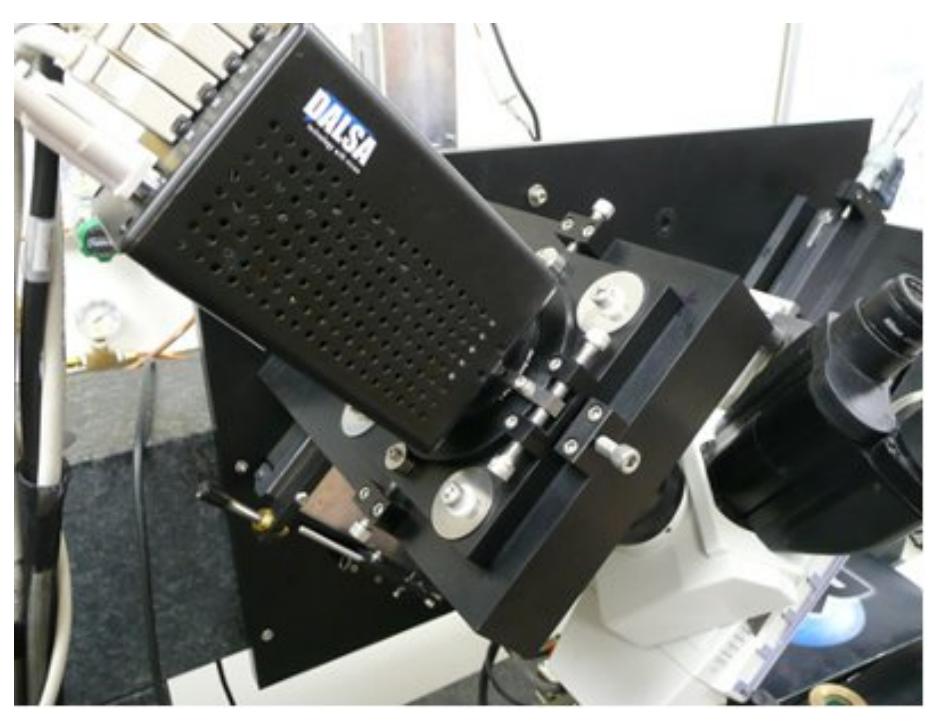

Figure 5. KESM camera and objective focusing controls.

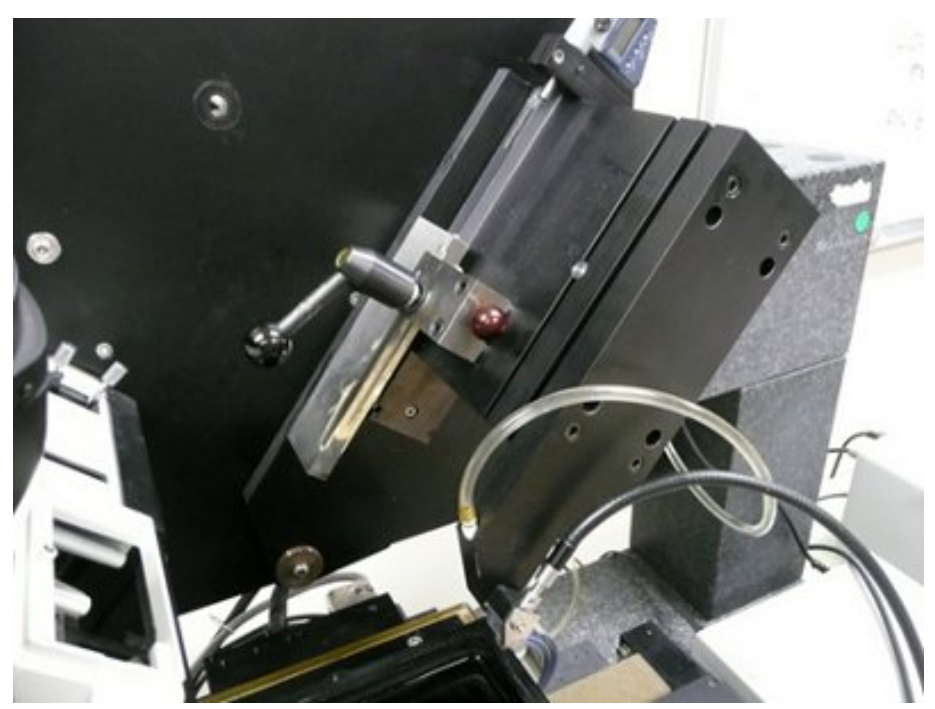

Figure 6. KESM knife assembly and controls. 


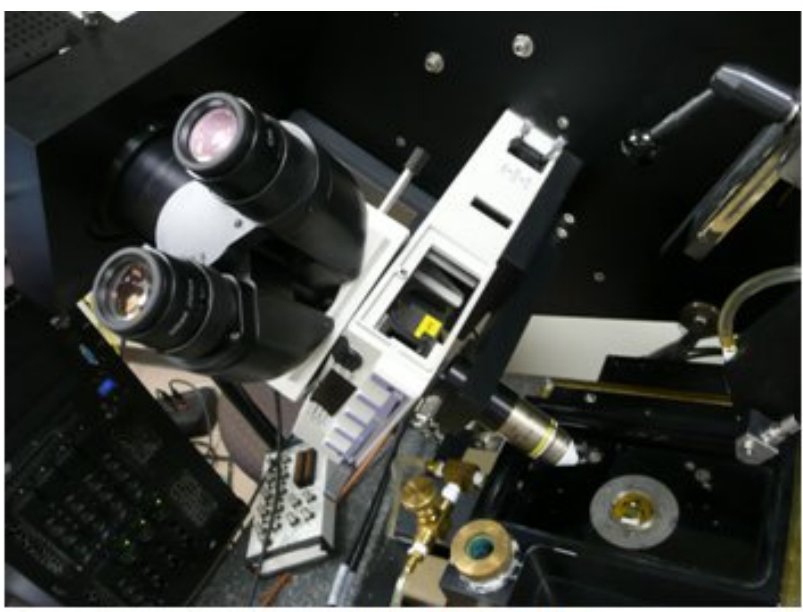

(a) Observation port
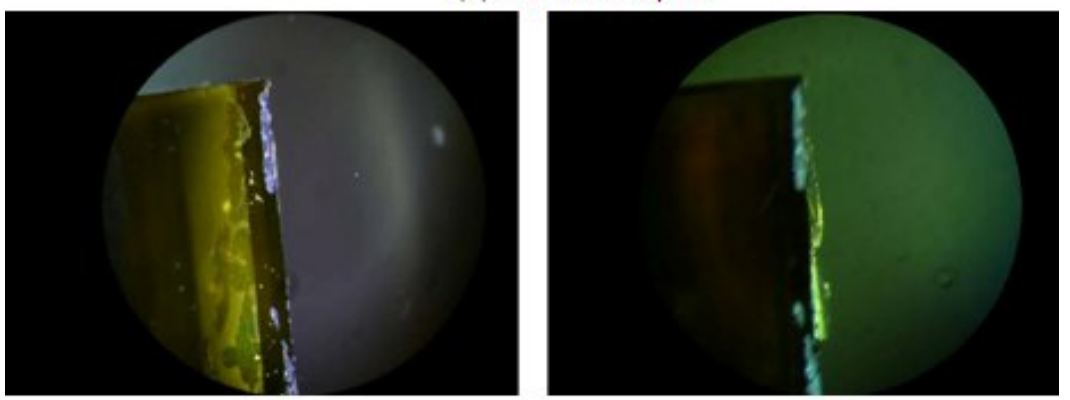

(b) In Focus (c) Out of focus

Figure 7. Initial focusing through the observation port.

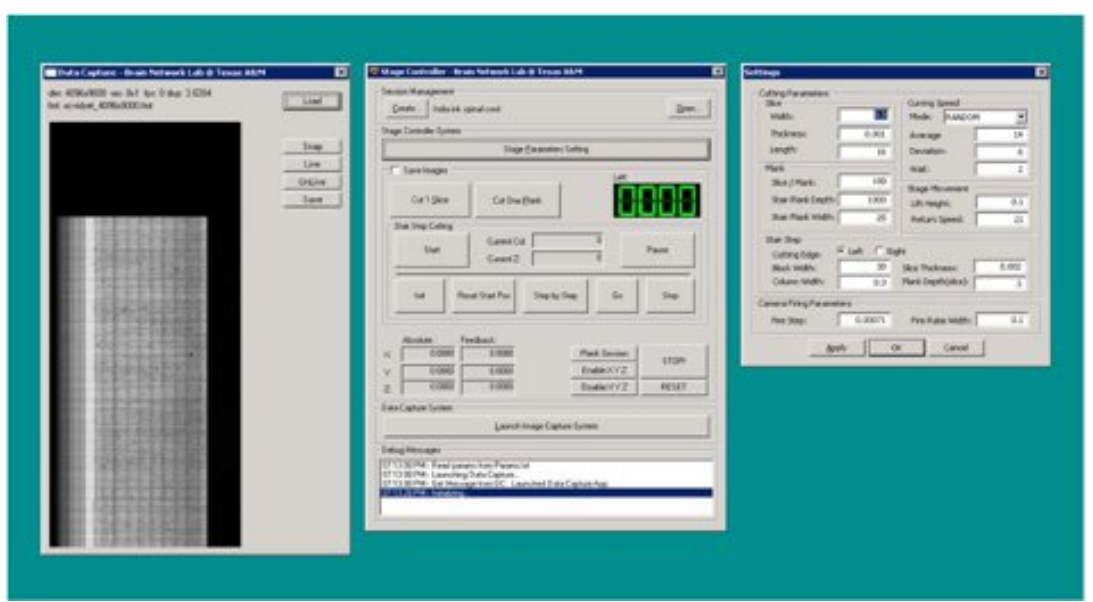

Figure 8. KESM Stage Controller 2 application (screenshot). 

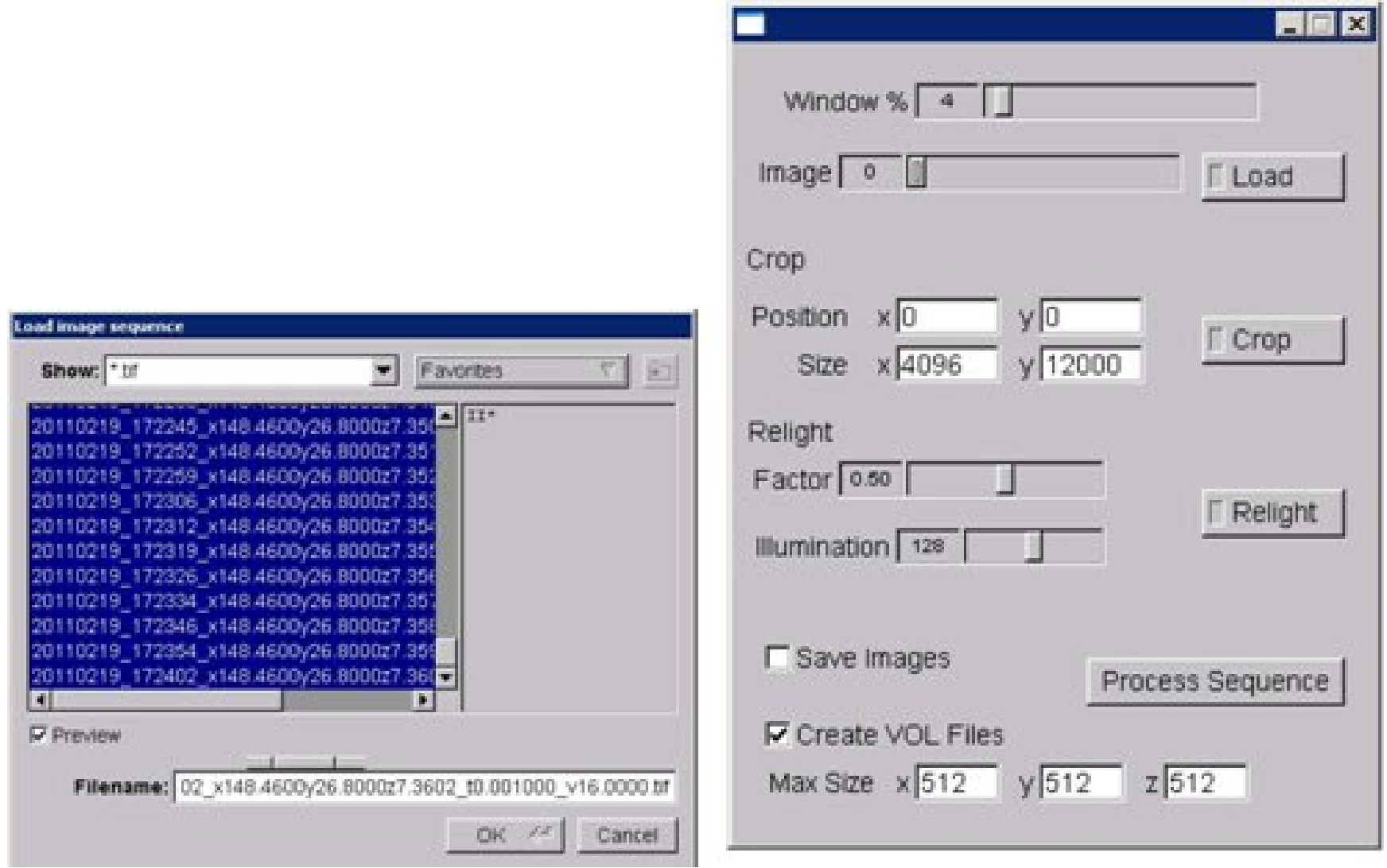

Figure 9. KESM stack processor application (screenshot).

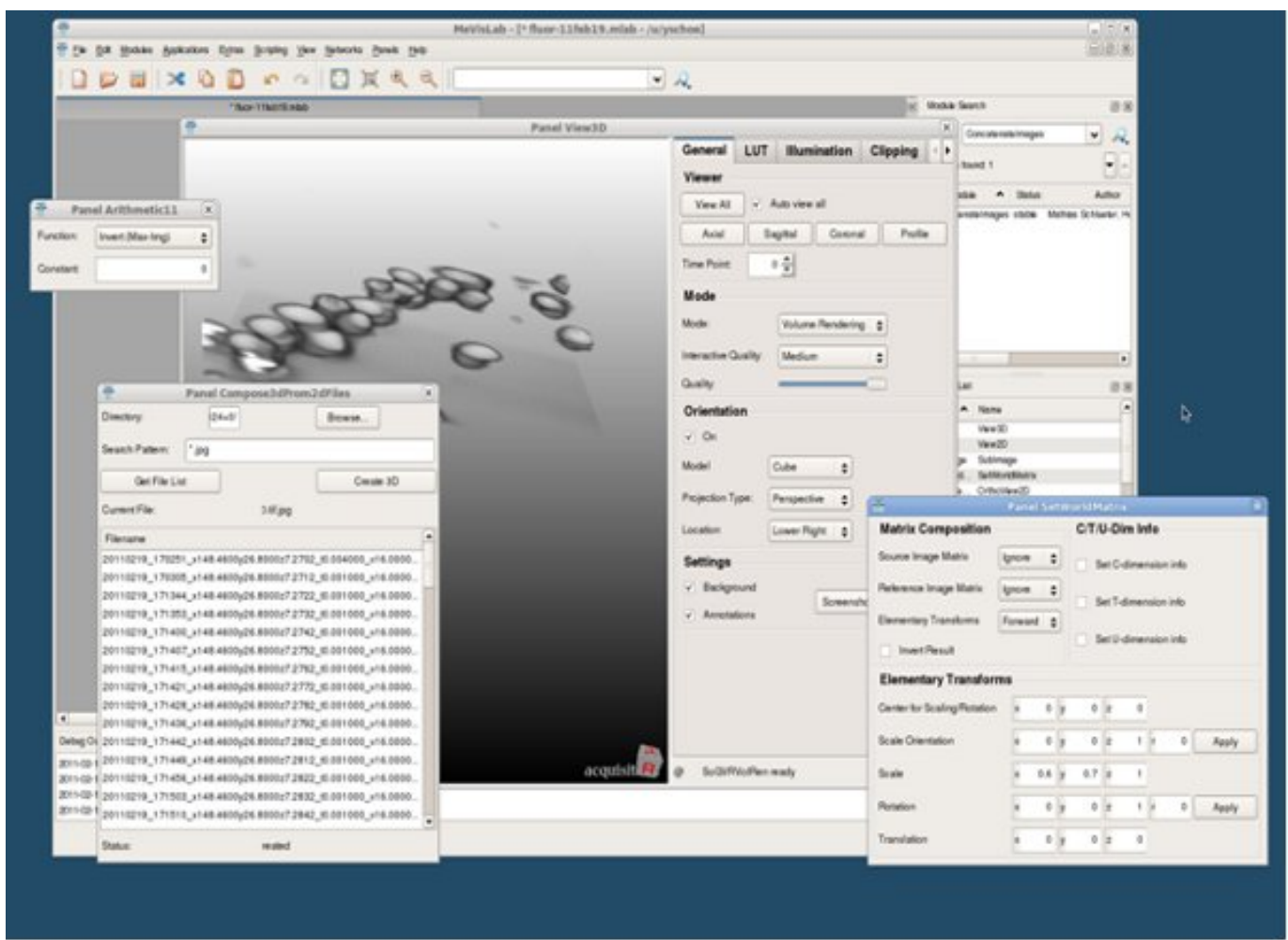

Figure 10. MeVisLab application (screenshot). 


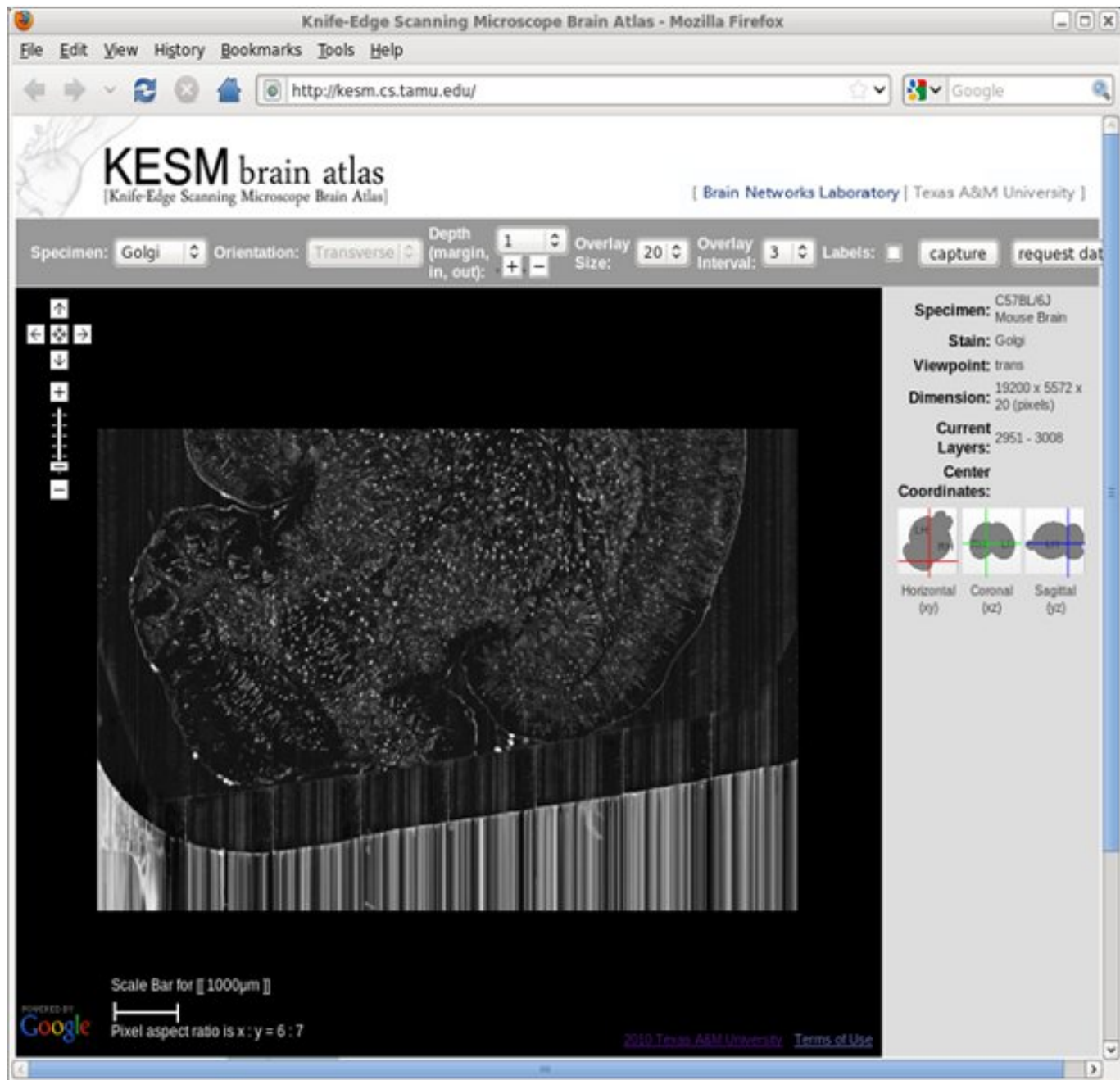

Figure 11. KESM Brain Atlas: Web-interface (screenshot). 

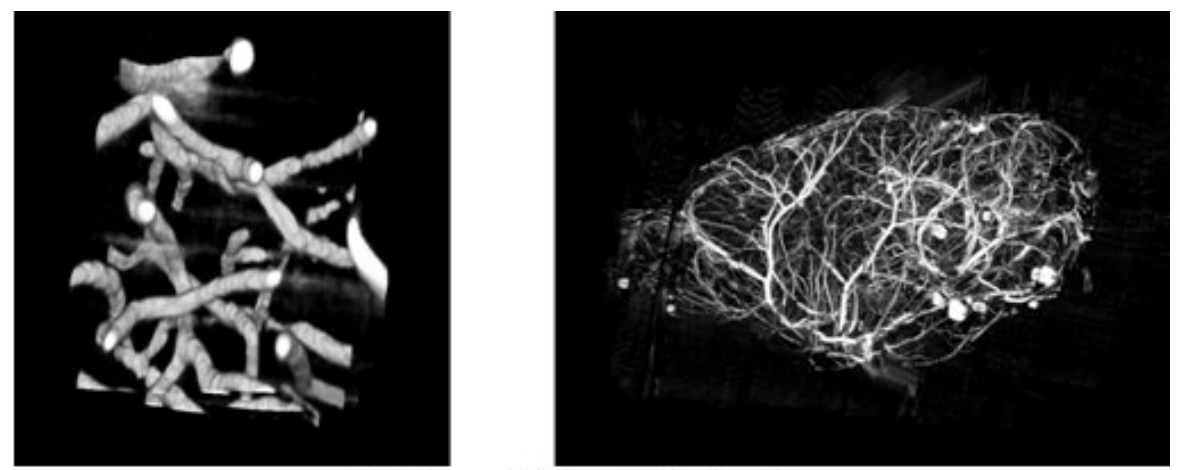

(a) Close-up (b) Saggital $(-A, \rightarrow P)$

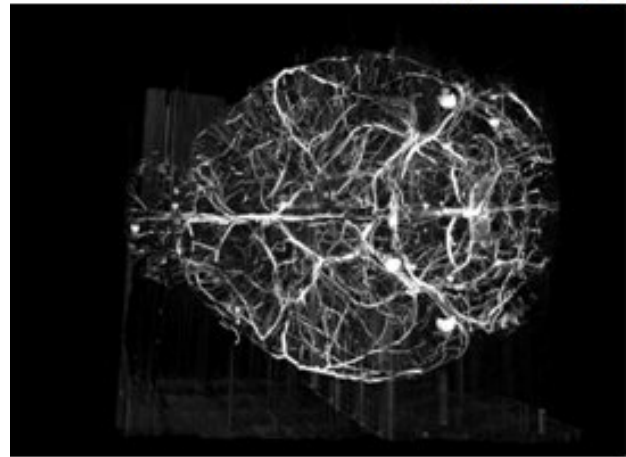

(c) Horizontal $(\leftarrow \mathrm{A}, \rightarrow \mathrm{P})$

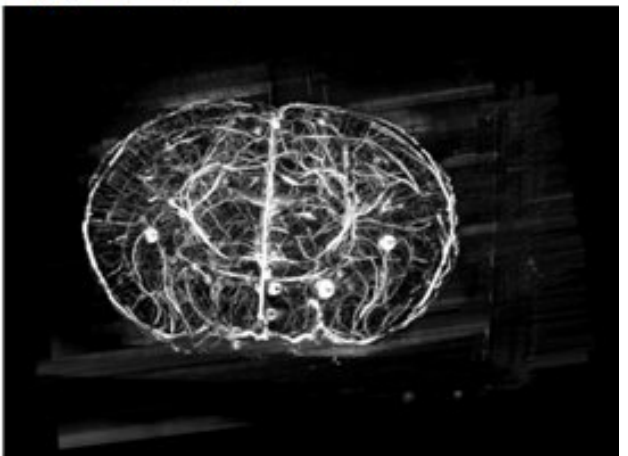

$(d)$ Coronal $(\uparrow \mathrm{D}, \downarrow \mathrm{V})$

Figure 12. KESM whole-brain India ink data. Volume visualizations of KESM data stacks are shown for the vascular data set. (a) Close-up of the vascular data. Width 100 um. (b-d) Three standard views of the whole mouse brain vasculature (subsampled from high-resolution data). Width $\sim 10 \mathrm{~mm}$. 

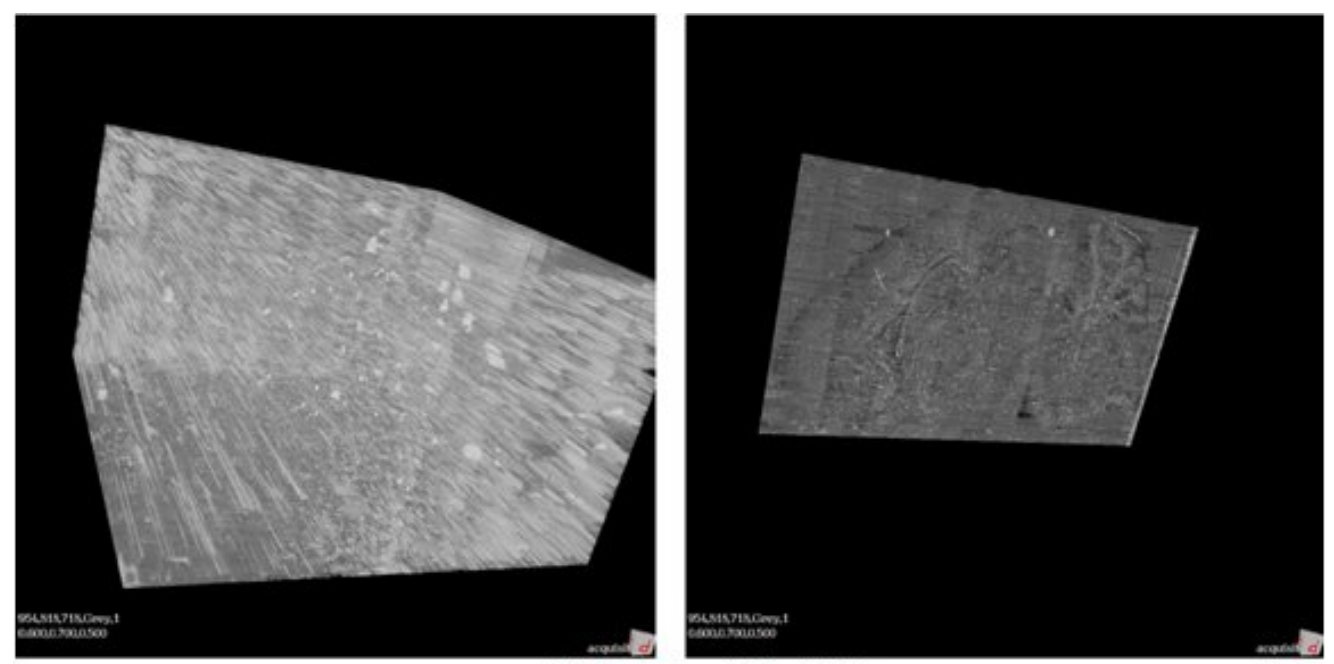

(a) Block view (b) Sagittal view

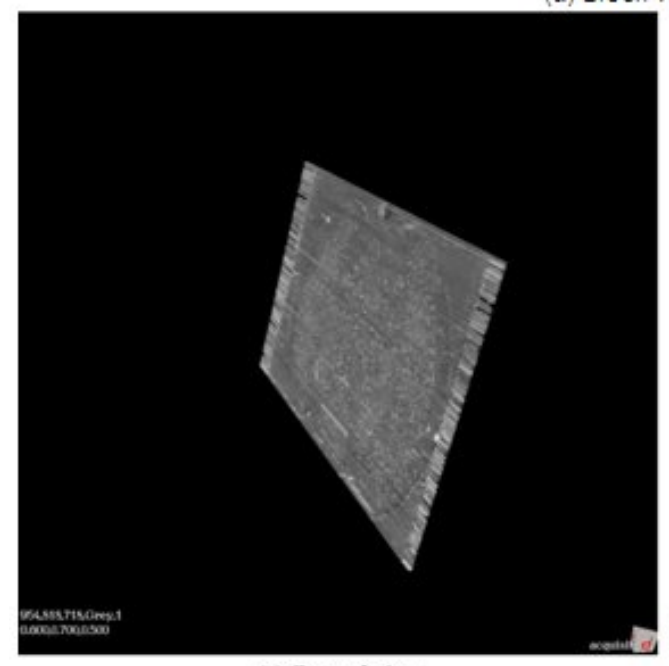

(c) Coronal view

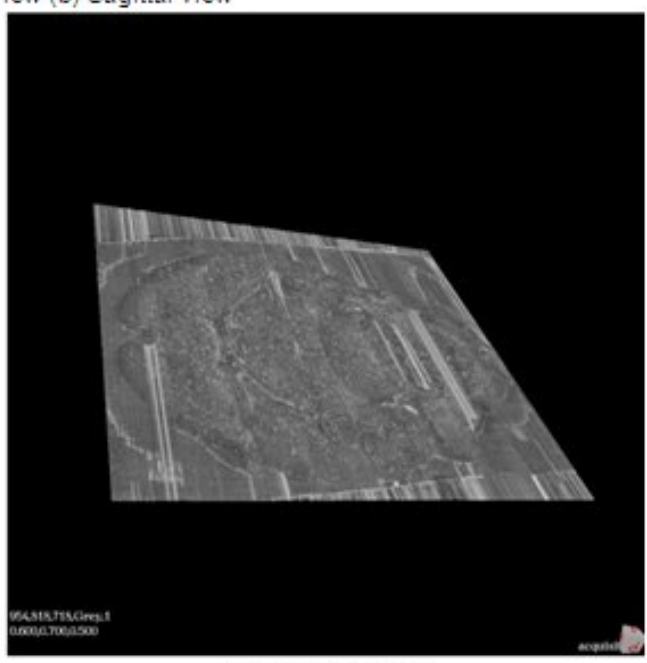

(d) Horizontal view

Figure 13. KESM whole-brain mouse Golgi data. 

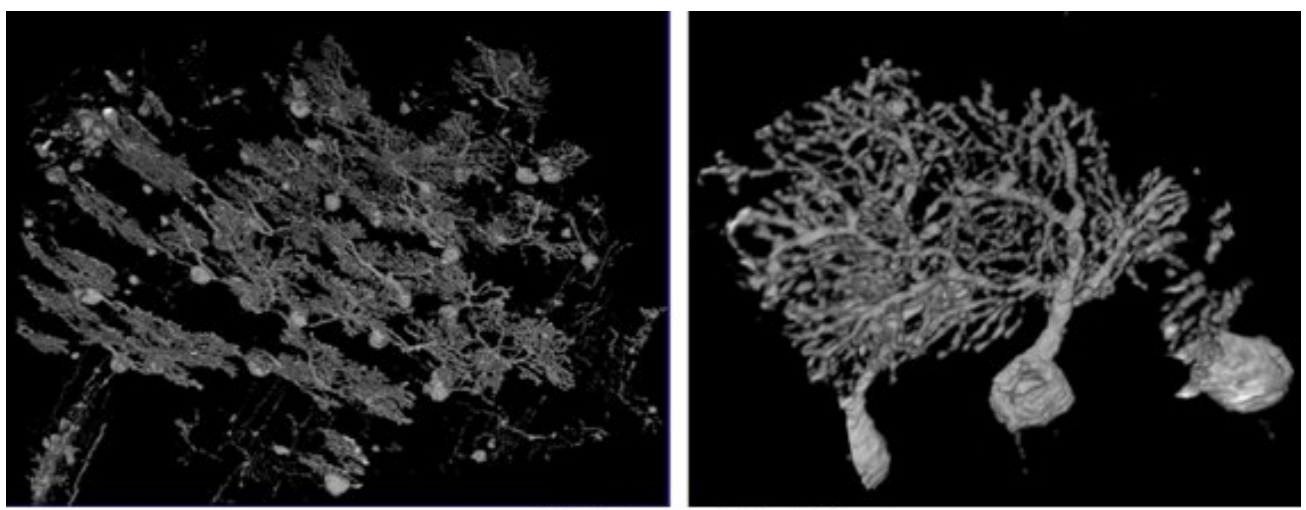

(a) Cerebellum (b) Purkinje cells

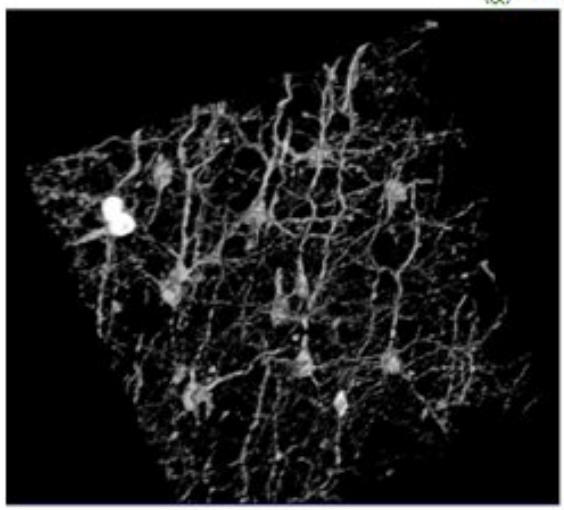

(c) Cortex

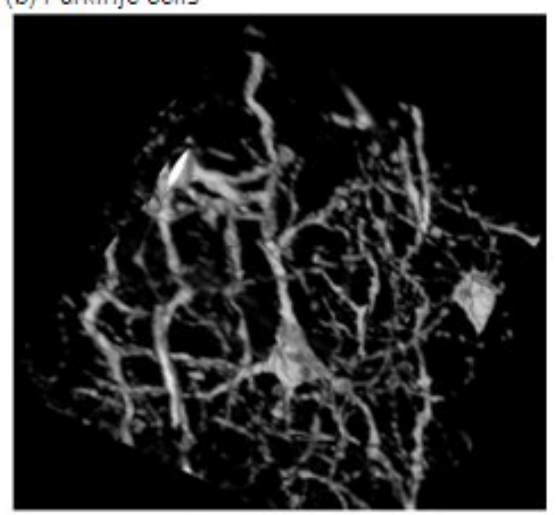

(d) Pyramidal cells

Figure 14. KESM Golgi data details. 

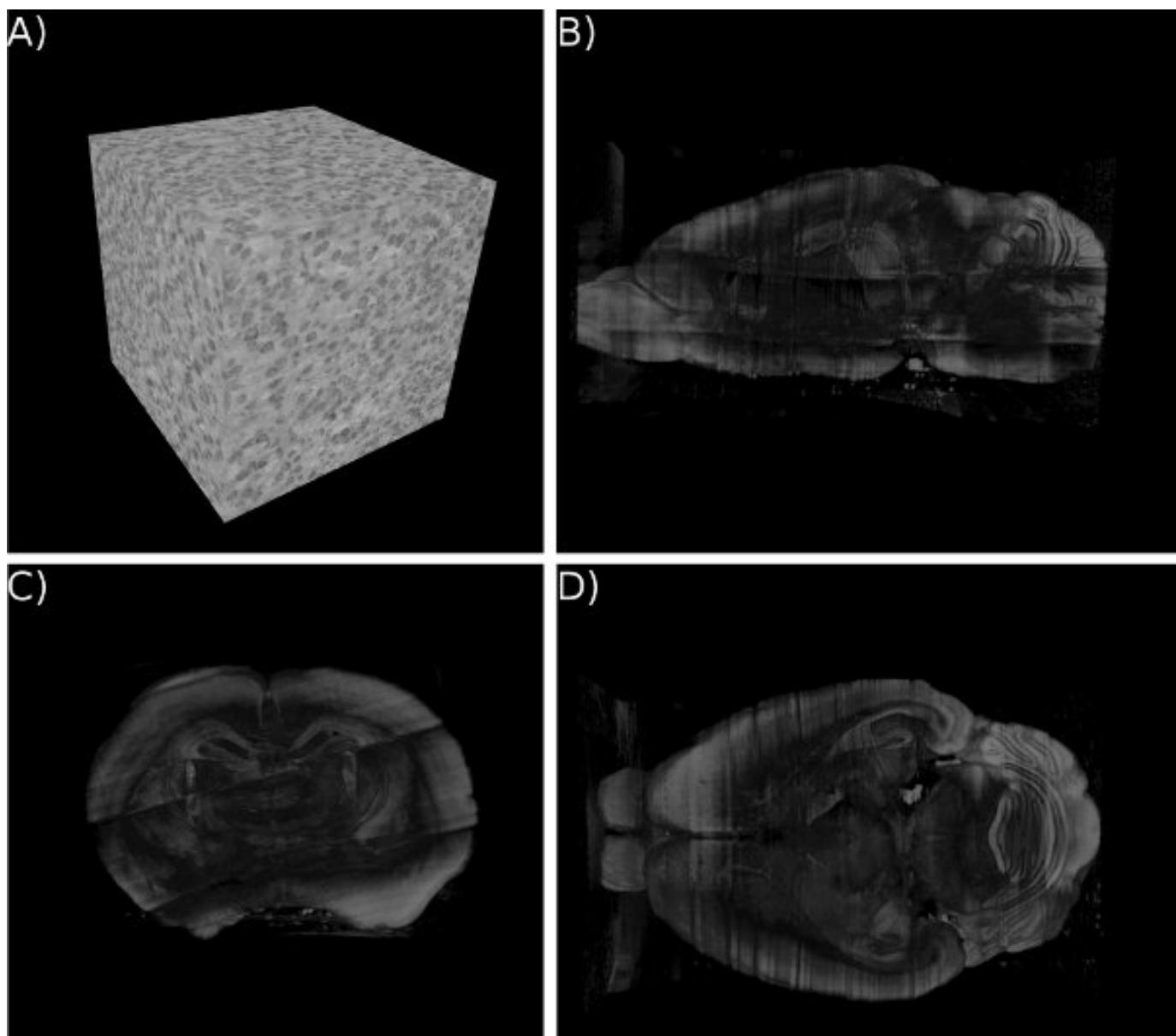

Figure 15. KESM whole-brain NissI data. (a) Close-up of the Nissl data. $\sim 300 \mathrm{um}^{3}$. (b-d) Three standard views of the whole mouse brain Nissl data (subsampled from high-resolution data). Cross section shown for a clear view of the internal structures.

\section{Discussion}

The KESM allows for a submicrometer-level survey of large volumes of biological specimen $\left(\sim 1 \mathrm{~cm}^{3}\right)$. This kind of volume is enough to hold whole small animal organs, such as brains, lungs, hearts, kidneys, etc. Scanned images from such organs can provide unprecedented quantitative information about the structural organization of these organs, and enable computational modeling of various functional aspects of these organs, including circuit and network dynamics, electrical properties, fluid and air flow dynamics, and muscular dynamics.

The specimen preparation protocol and post-analysis protocol detailed in this article are expected to help outside research groups to have easier access to the KESM and the resulting data. The KESM operation protocol will help these external researchers to appreciate the benefits and limitations of this unique imaging modality.

\section{Disclosures}

Todd Huffman is with 3Scan, a commercial company that manufactures and markets the KESM Technology (US patent \#6,744,572).

\section{Acknowledgements}

This project was funded by NIH/NINDS (\#1R01-NS54252), NSF (\#0905041,\#0079874), the Texas Advanced Technology Program (\#ATP-000512-0146-2001, \#ATP-000512-0261-2001), Texas A\&M Research Foundation, Department of Computer Science and Engineering at Texas A\&M University, and 3Scan. We would like to thank Bernard Mesa (Micro Star Technologies) for technical consultation and support for KESM instrumentation. Major parts of the design and implementation of the KESM was done by Bruce H. McCormick, who died in 2007.

\section{References}

1. Abbott, L.C. High-throughput imaging of whole small animal brains with the knife-edge scanning microscope. In Neuroscience Meeting Planner, Washington, DC: Society for Neuroscience Program No. 504.2 (2008). 
2. Abbott, L.C. \& Sotelo, C. Ultrastructural analysis of catecholaminergic innervation in weaver and normal mouse cerebellar cortices. Journal of Comparative Neurology, 426, 316-329, (2000).

3. Choe, Y., Abbott, L.C., Miller, D.E., Han, D., Yang, H.-F., Chung, J.R., Sung, C., Mayerich, D., Kwon, J., Micheva, K., \& Smith, S.J. Multiscale imaging, analysis, and integration of mouse brain networks. In Neuroscience Meeting Planner, San Diego, CA: Society for Neuroscience. Program No. 516.3. Online (2010).

4. Choe, Y., Han, D., Huang, P.-S., Keyser, J., Kwon, J., Mayerich, D., \& Abbott, L.C. Complete submicrometer scans of mouse brain microstructure: Neurons and vasculatures. In Neuroscience Meeting Planner, Chicago, IL: Society for Neuroscience Program No. 389.10. Online (2009).

5. Choe, Y., Abbott, L.C., Han, D., Huang, P.S., Keyser, J. Kwon, J., Mayerich, D., Melek, Z., \& McCormick, B.H. Knife-edge scanning microscopy: High-throughput imaging and analysis of massive volumes of biological microstructures. In A. Ravi Rao and Guillermo Cecchi, editors, High-Throughput Image Reconstruction and Analysis: Intelligent Microscopy Applications, Artech House, Boston, MA, 11-37 (2008).

6. Choe, Y., Abbott, L.C., Ponte, G., Keyser, J., Kwon, J., Mayerich, D., Miller, D., Han, D., Grimaldi, A.M., Fiorito, F., Edelman, D.B., \& McKinstry, J.L. Charting out the octopus connectome at submicron resolution using the knife-edge scanning microscope. Nineteenth Annual Computational Neuroscience Meeting: CNS²010, BMC Neuroscience., 11 (Suppl 1), 136, (2010).

7. Mayerich, D., Abbott, L.C., \& McCormick, B.H. Knife-edge scanning microscopy for imaging and reconstruction of three-dimensional anatomical structures of the mouse brain. Journal of Microscopy, 231, 134-143, (2008).

8. Mayerich, D., Kwon, J., Sung, C., Abbott, L.C., Keyser, J., \& Choe, Y. Fast macro-scale Transmission Imaging of Microvascular Networks Using KESM. Biomedical Optics Express, 2, 2888-2896, (2008).

9. McCormick, B.H. Development of the Brain Tissue Scanner, Technical Report, Department of Computer Science, Texas A \& M University, College Station, TX, March 18, 2002 [http://research.cs.tamu.edu/bnl/static/pubs/McC02.pdf], (2002).

10. Sporns, O., Tononi, G., \& Kötter, R. The human connectome: A structural description of the human brain. PLoS Computational Biology., 1, e42 (2005). 